\title{
Modelling electroluminescence in liquid argon
}

\author{
D.Y. Stewart ${ }^{b}$, G.J. Barker ${ }^{b}$, A.J. Bennieston ${ }^{b}$, P.F. Harrison ${ }^{b}$, P.K. Lightfoot ${ }^{a}{ }^{*}$ N. \\ McConkey $^{b}$, B. Morgan ${ }^{b}$, Y.A. Ramachers ${ }^{b}{ }^{\dagger}$ M. Robinson ${ }^{a}$, N.J.C. Spooner ${ }^{a}$ and L. \\ Thompson $^{a}$
}

${ }^{a}$ Dept. of Physics and Astronomy, University of Sheffield, Hicks Building, Hounsfield Road, Sheffield, S3 7RH, UK

${ }^{b}$ Dept. of Physics, University of Warwick, Coventry CV4 7AL, UK

\begin{abstract}
We present Monte-Carlo simulations of electron transport through liquid argon motivated by our recent observation of electroluminescence light emanating from a thick gaseous electron multiplier (THGEM) in a liquid argon volume. All known elastic and inelastic reaction cross-sections have been accounted for, providing electroluminescence light yield predictions for arbitrary electrostatic fields. This study concludes that the large field gradients needed to produce electroluminescence cannot be accounted for by straightforward electrostatic field calculations based on ideal THGEM holes, suggesting that further experimental investigations are required.
\end{abstract}

KEYWORDS: Noble-liquid detectors, electron multiplier (gas), THGEM, charge transport in liquid media, detector modelling and simulation II.

* current address: Dept. of Physics, University of York, York YO10 5DD, UK

${ }^{\dagger}$ Corresponding author; email: y.a.ramachers@warwick.ac.uk 


\section{Contents}

1. Introduction [1]

2. Monte-Carlo model 3

2.1 Kinematics $\quad$ 田

2.2 Cross sections

3. Results 6

3.1 Drift velocity 6

3.2 Transverse Diffusion Constant 6

3.3 Electroluminescence 8

4. Conclusion 9

\section{Introduction}

A recent experimental investigation [1] successfully demonstrated the proof-of-principle stage of a new technique for operating liquid argon time-projection chambers (LArTPC). Liquid argon technology is now rather mature, driven primarily by the ICARUS program [2] which has proven the principle of operation of a LArTPC for up to 600 ton mass. A detector capable of delivering the neutrino physics program of the future [3] will however need to be on a grander scale still with a fiducial mass of perhaps up to $100 \mathrm{kton}$.

The large step up in mass to 100 kton presents significant technological challenges, which have been addressed in recent years to a greater or lesser extent by a number of concept proposals: MODULAr [四] essentially stacks together many ICARUS modules to achieve the final volume placed in the CERN CNGS beam; GLACIER [5] drifts charge up to $20 \mathrm{~m}$ through a single liquid argon volume to be amplified and read-out in the gas directly above the liquid volume; FLARE [6] and LANNDD [7] are also based on a single volume of liquid argon but are internally segmented to limit the maximum charge drift distance and then read out using wire planes, similar to ICARUS. The latest project founded on the basis of a LArTPC detector using wire-plane readout is the ArgoNeuT project [8], currently taking data in the FNAL neutrino beam.

For most purposes the optimal detector design would feature a simultaneous tracking and homogeneous calorimetry target with low momentum threshold and high efficiency and with the potential of large active volumes. This technology would be especially relevant to the LAGUNA [9] proton decay and neutrino physics project, upgrades to the existing long baseline T2K experiment [10], and the proposed neutrino factory project [11].

The existing concepts can be classified as either single-phase or double-phase LArTPCs. The main conceptual difference apart from the number of argon phases utilised is the gain. Single-phase 
devices do not show any internal gain and therefore involve small signal-to-noise ratios and rely on sensitive front-end electronics. Such a readout technology is limited with respect to the wire-plane (or anode) readout area that is practically achievable and costly due to the large number of readout channels required. The second technology, double-phase readout, benefits from internal gain due to signals in the gas-phase where avalanches in argon can increase the primary signal substantially. The price for this advantage is the restriction to gas-readout which enforces either extremely long drift lengths or very large surface area (shallow tank).

The recent proof-of-principle demonstration in [1] paves the way for a third alternative liquid argon readout technology. It combines the separate advantages of the above types of LArTPCs by using single-phase operation with an amplified signal. The idea is to utilise the robust and mature single-phase TPC concept and implement optical readout of light produced by electroluminescence in liquid argon.

The new concept of operating a Thick Gas Electron Multiplier (THGEM) [12] directly in the liquid and utilising electroluminescence for optical readout, i.e. light emitted in the THGEM holes with, for example, silicon photomultipliers (SiPM) readout [13], presents the possibility of fine grain tracking (spatial resolution of order $\mathrm{mm}$ ) with high signal-to-noise ratio. This technology uses only robust low-cost components directly in the liquid volume (see the sketch of the set-up in Fig. (1). The THGEM provides an excellent imaging plane for electroluminescence. Electrons initially released by ionisation in the fiducial volume drift towards the THGEM holes, where the presence of strong electric fields inside the holes results in well-localised light sources.

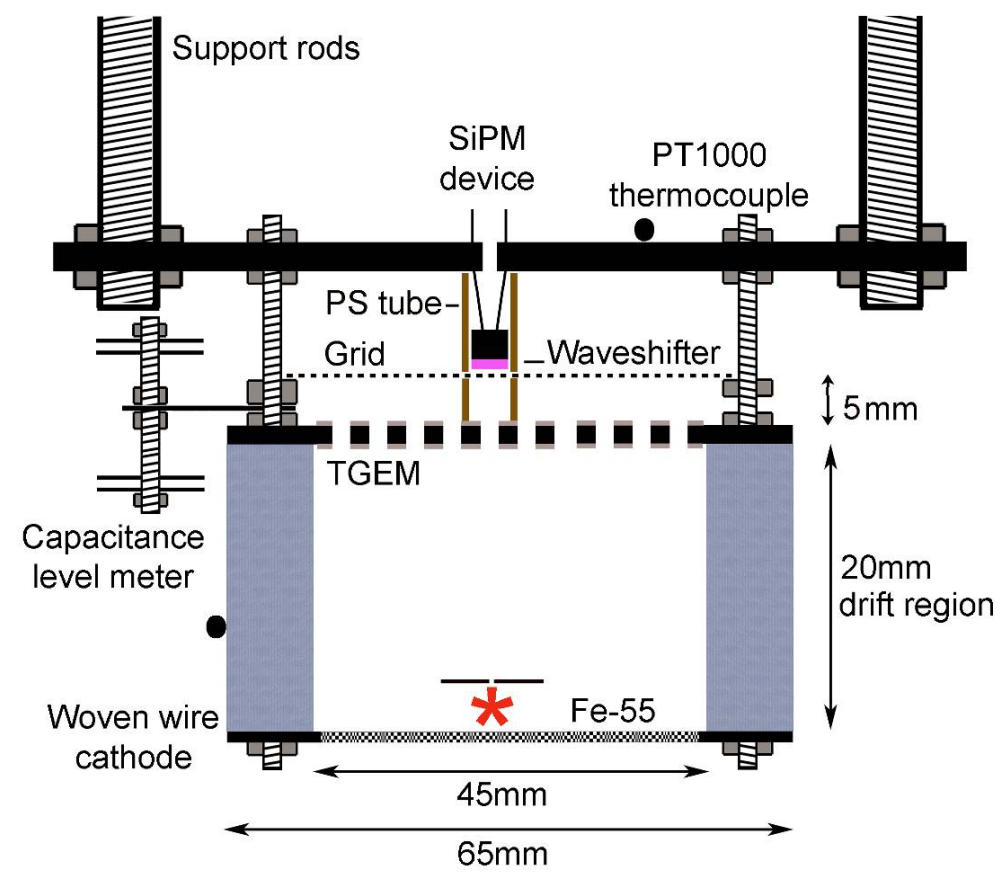

Figure 1. Sketch of the experimental setup. The THGEM element is a double-clad printed circuit board with regularly spaced, drilled holes, confining strong electrostatic fields when a bias is applied between the THGEM sides. 'PS' stands for polystyrene. More details can be found in [U].

This article focuses on creating and testing an essential tool for model building to understand 
the electroluminescence observations taken during the proof-of-principle experiment, thus guiding future measurements and optimisations. It is expected that the main impact of such a model would be to optimise THGEM geometry and to improve and explain the weak energy resolution obtained in [四]. The following section introduces the Monte-Carlo model which describes drifting electrons in liquid argon at arbitrary electric field strengths, before the results are presented in the subsequent section.

\section{Monte-Carlo model}

Our Monte-Carlo simulation follows previous publications on simulations of electron transport in gaseous argon [14]. Before discussing the details of the simulation it is useful to note a few estimates of what can be expected when transporting electrons in liquid argon. Given an atomic number density of $2.12 \times 10^{22} \mathrm{~cm}^{-3}$ [15] and a representative electron/argon cross section at low energies of $5.5 \times 10^{-16} \mathrm{~cm}^{2}$ [16] [17] the mean free path would be about $L=0.86 \mathrm{~nm}$. Hence modelling the transport of such an electron over any appreciable distance such as in modern detectors is numerically quite challenging. Such a length is traversed by a thermal electron (an electron with $\sim 0.012 \mathrm{eV}$ kinetic energy at LAr temperatures) in a time $\tau \sim 10^{-14} \mathrm{~s}$. This length scale also enables an estimation of the electric field strength, $E$, needed to accelerate an electron to kinetic energies, $T$, namely $E \sim 10 T \mathrm{MV} / \mathrm{cm}$ with $T$ in $\mathrm{eV}$.

The expected behaviour of an electron of a few $\mathrm{meV}$ in a dense medium such as liquid argon can be described as a dynamic particle with speeds of the order of $10^{6} \mathrm{~m} / \mathrm{s}$ moving in a random walk due to collisions with atoms. Such high speeds are necessary to explain energy dependent processes like inelastic scattering which occur at above $10 \mathrm{eV}$ energies (corresponding to speeds of more than $1.8 \times 10^{6} \mathrm{~m} / \mathrm{s}$ for an electron). The average motion, however, yields a relatively slow drift velocity in the direction of the electric field and an additional diffusion motion in the transverse directions.

Our chosen Monte-Carlo method follows other simulations of charged particle transport in electric fields and is referred to as a null-collision method or self-scattering[18] [19]. Both references provide detailed independent explanations of the method which found further application in [16], [20], [21] and [22]. This method is based on adding an artificial cross section for 'null' collisions, $\sigma_{\text {null }}$, which do not affect the particle in any way, in order to saturate an upper bound on the (energy-dependent) cross-section $\sigma$. Following [23], a slightly changed null-collision method compared to the original in [18] has been applied. Thus a constant sum of velocity-weighted cross sections $v \sigma+v \sigma_{\text {null }}=K_{\max }$ [23] is created, with $K_{\max }$ a constant and $v$ the particle velocity. This has the conceptual benefit of enabling the method to draw random collision times from a single exponential distribution with the mean value $\tau=1 /\left(n K_{\max }\right)$ [23], with $n$, the argon number density. The probability of real collisions occurring compared to null collisions is then simply the ratio $v \sigma / K_{\max }$. The constant $K_{\max }$ is chosen large enough to accommodate all possible real cross sections and velocities such that the ratio is always less than one. Given this, the value of the constant has no influence on the simulation results. 


\subsection{Kinematics}

The fractional kinetic energy retained in energy transfer collisions is implemented as

$$
\frac{T_{e}^{\prime}}{T_{e}}=1-4 \frac{m_{e} m_{A}}{\left(m_{e}+m_{A}\right)^{2}} \cos ^{2} \phi
$$

where $\mathrm{T}_{e}$ denotes the electron kinetic energy before and $\mathrm{T}_{e}^{\prime}$ after the scattering event while $\mathrm{m}_{e}$ and $\mathrm{m}_{A}$ are the electron and argon rest masses, respectively. The argon recoil angle $\phi$, is given approximately by $\sin 2 \phi \approx \sin \theta$ with $\theta$ the electron scattering angle. This angle $\theta$ is sampled randomly from the normalised differential cross section at the corresponding energy, see section 2.2. The large mass mismatch between the electron and the argon atom ensures that these energy transfers per reaction are small, but they are important because of the relatively high frequency of interactions.

\subsection{Cross sections}

Electron-argon cross sections implemented in the simulation are summarised in Fig. 2. Scattering cross sections are seen to dominate over excitation/ionisation processes which turn-on for electron energies above about $10 \mathrm{eV}$. The simulation decides on which process to model according to a comparison of the cross section ratio to a uniformly distributed unit random variable.

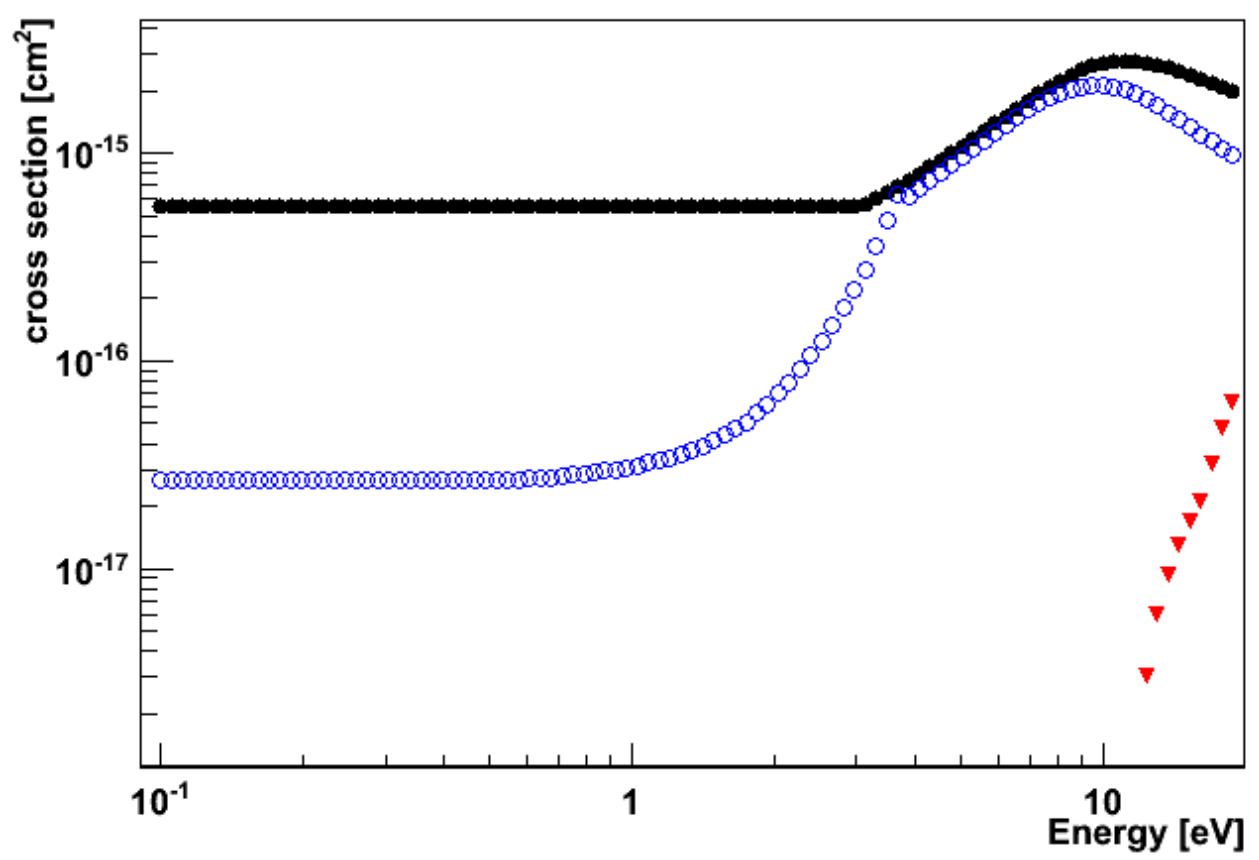

Figure 2. Cross sections implemented in the simulation for electron-argon interactions as a function of the electron energy. Scattering processes are divided into energy transfer cross sections $\sigma_{E}$ (filled circles) and momentum transfer cross sections $\sigma_{M}$ (open circles). The triangle symbols represent excitation/ionisation cross section measurements. For a discussion and references, see text. 


\begin{tabular}{rrrrr}
\hline $\mathrm{p}_{0}$ & $\mathrm{p}_{1}$ & $\mathrm{p}_{2}$ & $\mathrm{p}_{3}$ & $\mathrm{p}_{4}$ \\
\hline 0.04734 & -0.012248 & 0.035742 & -0.023278 & 0.008771 \\
\hline
\end{tabular}

Table 1. Fourth order polynomial fit parameter $\mathrm{p}_{i}, S(\varepsilon)=\sum_{i=0}^{4} p_{i} \varepsilon^{i}$, for the liquid argon structure function, $\mathrm{S}(\varepsilon)$, defined for energies $\varepsilon$ between $0.5 \mathrm{eV}$ and $3.75 \mathrm{eV}$. For more details, see text.

An important concept in the simulation of electron scattering in media is the distinction between energy transfer collision reactions, which only change the magnitude of the velocity, and momentum transfer, which change only the direction of the velocity vector leaving the magnitude identical. The distinction between these two types of collisions proved to be a cornerstone to the understanding of electron transport in condensed noble gases [24], [17] and both processes have been assigned separate cross sections as advocated in [25], [24]. The distinction between momentum transfer $\left(\sigma_{M}\right)$ and energy transfer $\left(\sigma_{E}\right)$ cross sections is implemented in the simulation via the concept of a structure function $\mathrm{S}(\varepsilon)$ [24], [17] ( $\varepsilon$ here is the electron kinetic energy), where $\sigma_{M}=\sigma_{E} S(\varepsilon)$.

Below $0.5 \mathrm{eV}$, according to the model by [24], the behaviour of the energy transfer cross section, $\sigma_{E}$, changes compared to the gas case - due to collective effects related to the higher atomic density. The cross section and structure function are predicted to become essentially constant and we have implemented the following values for liquid argon: $\sigma_{E}=5.5 \times 10^{-16} \mathrm{~cm}^{2}$ [16], [117] and $S(0)=0.048$ [24]. Additionally, differential cross sections are assumed to be isotropic [17]].

Above $0.5 \mathrm{eV}$ electron kinetic energy, $\sigma_{E}$ is expected to remain constant but the structure function begins to increase. Analytical expressions for the structure function are given in the literature but turn out to be relatively complex, particularly for a computationally intensive application. Instead we have implemented a parameterisation of the function based on the results in [26]. A fourth order polynomial fit was performed which reproduced the data with discrepancy less than $2 \%$ between the energies of $0.5 \mathrm{eV}$ and $3.75 \mathrm{eV}$. The fit and parameter values are presented in Tab. [1.

This structure function parameterisation and the assumption that $\sigma_{E}$ is constant, as advocated in [17], is implemented for the electron kinetic energy range between $0.5 \mathrm{eV}$ and $3.07 \mathrm{eV}$. At energies higher than this however, as discussed in [17], it was assumed that the scattering cross sections for liquid argon should follow those valid for argon gas. Therefore for energies up to $3.75 \mathrm{eV}$ the structure function parameterisation was implemented together with gas cross sections from [27]. For energies higher than $3.75 \mathrm{eV}$ it is assumed that the structure function approach plays no role. Energy and momentum transfer cross sections (both absolute and differential) valid for gas were implemented from [27].

Finally, excitation/ionisation reactions have to be taken into account. The excitation reaction is the inelastic reaction responsible for electroluminescence in argon. For the following, excitation cross sections yielding electroluminescence photons are of primary interest. The cross section data and available calculations are far more uncertain compared to the scattering processes. Cross sections have been implemented according to values presented in [28] and have been parameterised by a sixth order polynomial, valid from a threshold of $11.55 \mathrm{eV}$ to $50 \mathrm{eV}$ (see Tab. 目, Fig. 2). 


\begin{tabular}{rrrrrrr}
\hline $\mathrm{p}_{0}$ & $\mathrm{p}_{1}$ & $\mathrm{p}_{2}$ & $\mathrm{p}_{3}$ & $\mathrm{p}_{4}$ & $\mathrm{p}_{5}$ & $\mathrm{p}_{6}$ \\
\hline 34.7531 & -10.2377 & 1.10632 & -0.0562047 & 0.00147617 & $-1.94974 \mathrm{e}-5$ & $1.02707 \mathrm{e}-7$ \\
\hline
\end{tabular}

Table 2. Sixth order polynomial fit parameter $\mathrm{p}_{i}, \sigma_{\text {excitation }}\left[\mathrm{m}^{2}\right]=10^{-21} \sum_{i=0}^{6} p_{i} \varepsilon^{i}$, for the electron excitation cross section in liquid argon, defined for energies $\varepsilon$ between threshold, $11.55 \mathrm{eV}$, and $50 \mathrm{eV}$, using data from [28].

\section{Results}

In order to test the quantitative predictions of the simulation, we present below results for drift velocities and diffusion of electrons in liquid argon and compare to existing data. Furthermore we present predictions for electroluminescence in liquid argon and comment on the agreement with data.

\subsection{Drift velocity}

The results on electron drift velocity in liquid argon are shown in Fig. B. The general shape of the relation between drift velocity and electric field strength compares well with independent calculations [20], [17] and measurements [29], and references in [17], for field strengths below a few $10^{6}$ $\mathrm{V} / \mathrm{m}$. The predicted plateau in drift velocity for field strengths higher than this does not seem to be supported by the data [30] and has been highlighted as a challenge to theory in several publications. In [17] this phenomenon is traced back to the exponentially rising slope of the momentum transfer cross section illustrated in Fig. 因.

\subsection{Transverse Diffusion Constant}

Diffusion constants have been extracted from the simulation based on a fixed drift field strength of $2 \mathrm{kV} / \mathrm{cm}$ in order to aid comparison with existing calculations and measurements. Following [31], we quantify transverse diffusion as: $\left\langle r_{R M S}^{2}\right\rangle=4 D_{\perp} T$, where $\left\langle r_{R M S}^{2}>\right.$ is the mean variance of electron trajectories transverse to the drift direction, $D_{\perp}$ is the transverse diffusion constant and $\mathrm{T}$ the total time of electron motion (or using the drift velocity, one might use the total drift length here). The factor four results from two independent degrees of freedom of motion.

Results for diffusion in liquid argon are scarce. Analytical calculations exist [17], [32] and lead to the following expected range valid for a drift field of $2 \mathrm{kV} / \mathrm{cm}: 14 \mathrm{~cm}^{2} / \mathrm{s} \leq D_{\perp} \leq 17 \mathrm{~cm}^{2} / \mathrm{s}$ (where we have translated the published predictions of characteristic energy into diffusion constants). This range is compatible with the measurements of characteristic energy from [31] at their lowest measured drift field strength of $2 \mathrm{kV} / \mathrm{cm}$. Our study, presented in Fig. $甘$, is based on 100 simulated electron trajectories each with a fixed start point which is the origin of the Cartesian coordinate system with zero initial velocity. A Gaussian fit to the majority of the diffusion constants results in a value of $D_{\perp}=13.9 \pm 6.0 \mathrm{~cm}^{2} / \mathrm{s}$. As seen in Fig. 1 , this excludes about one third of the total number of simulated trajectories which constitute a tail to higher values. These larger values seem to be caused by trajectories that have not fully reached an uncorrelated state after the artificial termination of the simulation imposed after $2 \times 10^{9}$ collisions, corresponding to a simulated drift time of about $11.4 \mu \mathrm{s}$. Similar challenges to diffusion constant simulations have been pointed out previously in [33] and have been traced back to steep changes in cross sections at low energies. 


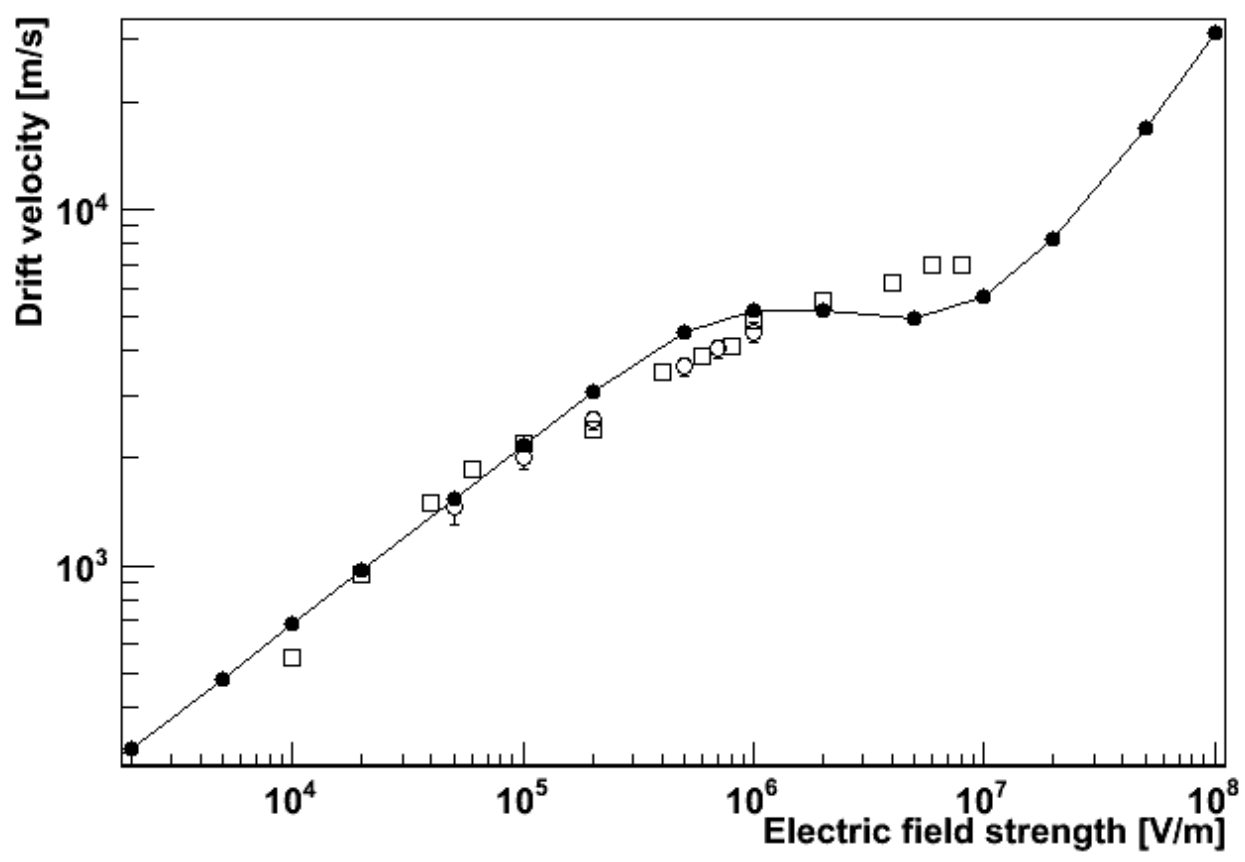

Figure 3. Simulated electron drift velocity in liquid argon as a function of electric field strength (black dots along line), compared to recent measurements from [29] (hollow circles) and older data from [30] (hollow squares). For details, see text.

Any experiment able to measure diffusion will actually measure the spread of the electron cloud rather than each single electron trajectory. The only existing measurement [B1] indicated far smaller transverse variances of electron clouds compared to our result - see top panel of Fig. $\rightarrow$ which corresponds to a transverse diffusion constant of $71 \mathrm{~cm}^{2} / \mathrm{s}$ (transporting together the 100 electrons illustrated in Fig. (4). Attempting to reconcile measurements with the simulation revealed (as was seen for drift velocities), a strong dependence of the diffusion constant on the size of the (constant) momentum transfer cross section at low electron energies. In [31] the authors suggest a cross section of between 3 to $4 \times 10^{-17} \mathrm{~cm}^{2}$ from their characteristic energy measurement, which is higher than the value assumed in the present study. Changing our momentum transfer cross section artificially to a value of $3.5 \times 10^{-17} \mathrm{~cm}^{2}$ (a $32 \%$ increase) changes the diffusion calculations drastically. The cloud diffusion constant drops to a more acceptable value of around $26 \mathrm{~cm}^{2} / \mathrm{s}$, i.e. by almost a factor of a third, and individual electron trajectory diffusion to a mean value of 9.2 $\mathrm{cm}^{2} / \mathrm{s}$ with much reduced spread even for shorter simulated trajectories. However, the drift velocity drops likewise to almost half its previous value at $2 \mathrm{kV} / \mathrm{cm}$.

A more satisfactory modelling of diffusion may come from the observation made in [17] where it is pointed out that the transverse diffusion constant depends crucially on lower energy electrons with fast electrons from the upper tail of the energy spectrum contributing little to diffusion. A slightly increasing slope towards low energies for the cross section may be able to account for reconciling the drift velocity data while simultaneously keeping the size of diffusion constants in 
check. To understand further the simulation of these effects is a work in progress and to this end, new measurements of transverse diffusion constants at lower fields would be very useful.

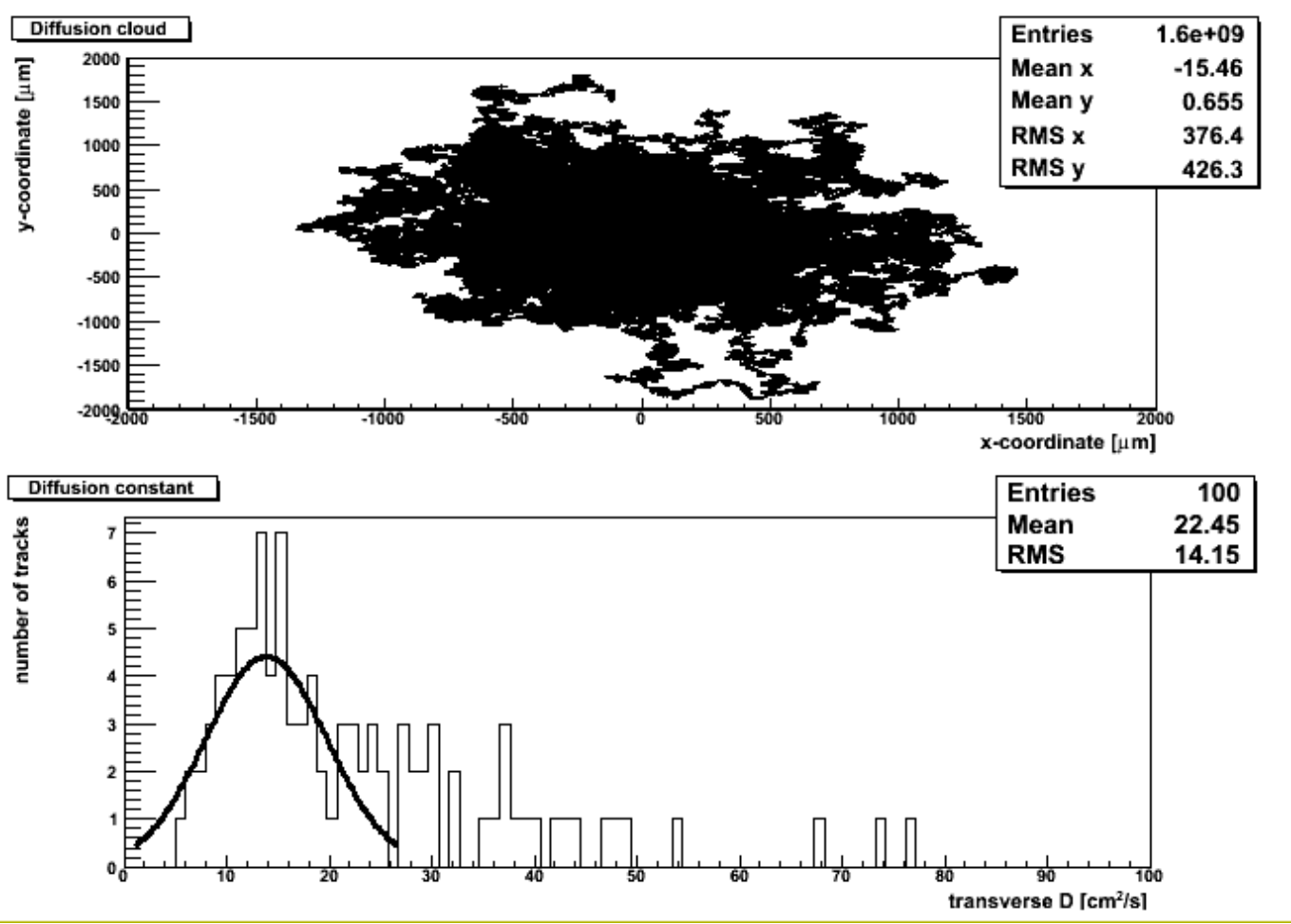

Figure 4. The upper panel shows the diffusion cloud of 100 individually simulated electrons after drifting through liquid argon in a drift field of $2 \mathrm{kV} / \mathrm{cm}$. The statistical measures of the cloud are indicated in the insert, such as the RMS values and the slightly off-centre mean values. Taking this ensemble of electrons for transverse diffusion constant calculations results in a value of $71 \mathrm{~cm}^{2} / \mathrm{s}$ as discussed in the text (the number of entries reflects the summing of 100 histograms on a $4000 \times 4000$ bin histogram, not the total of $2 \times 10^{11}$ collision events displayed in it). The lower panel shows the histogram of individually calculated transverse diffusion constants for each simulated electron. About two thirds of electrons form a Gaussian peak of diffusion constant values at $(13.9 \pm 6.0) \mathrm{cm}^{2} / \mathrm{s}$, the rest displaying significantly higher diffusion constant values, presumably due to still correlated motion, see text.

\subsection{Electroluminescence}

Electroluminescence measurements in Argon gas have been presented in [34] where it was found that a roughly linear relationship exists between the reduced electroluminescence yield $(Y / N)$ and the reduced electric field gradient $E / N$ - where the normalisation factor $N$ is the number density of the gas. For comparison to this result, we present in Fig. 5 the $Y / N$ vs $E / N$ prediction for liquid argon from our simulation. The data in Fig. 5 have been fitted to a third order polynomial whose parameters are given in Tab. 3. These light yields for the liquid state are comparable to yields in gas from [34] when taking into account the required [[1] higher electric field and the roughly 800-fold increase in density for the liquid. This result is compatible with direct observations from [1] where 


\begin{tabular}{rrrr}
\hline $\mathrm{p}_{0}$ & $\mathrm{p}_{1}$ & $\mathrm{p}_{2}$ & $\mathrm{p}_{3}$ \\
\hline-0.0436844 & $1.93051 \mathrm{e}-03$ & $-2.89617 \mathrm{e}-05$ & $1.4795 \mathrm{e}-07$
\end{tabular}

Table 3. Third order polynomial fit parameter $\mathrm{p}_{i}, Y / N=\sum_{i=0}^{3} p_{i}(E / N)^{i}$, for the empirical fit to the yield relation. For more details, see text.

similar electroluminescence gain factors (photons produced per drifting electron) are measured once sufficiently high electric fields are reached in order to initiate the process.

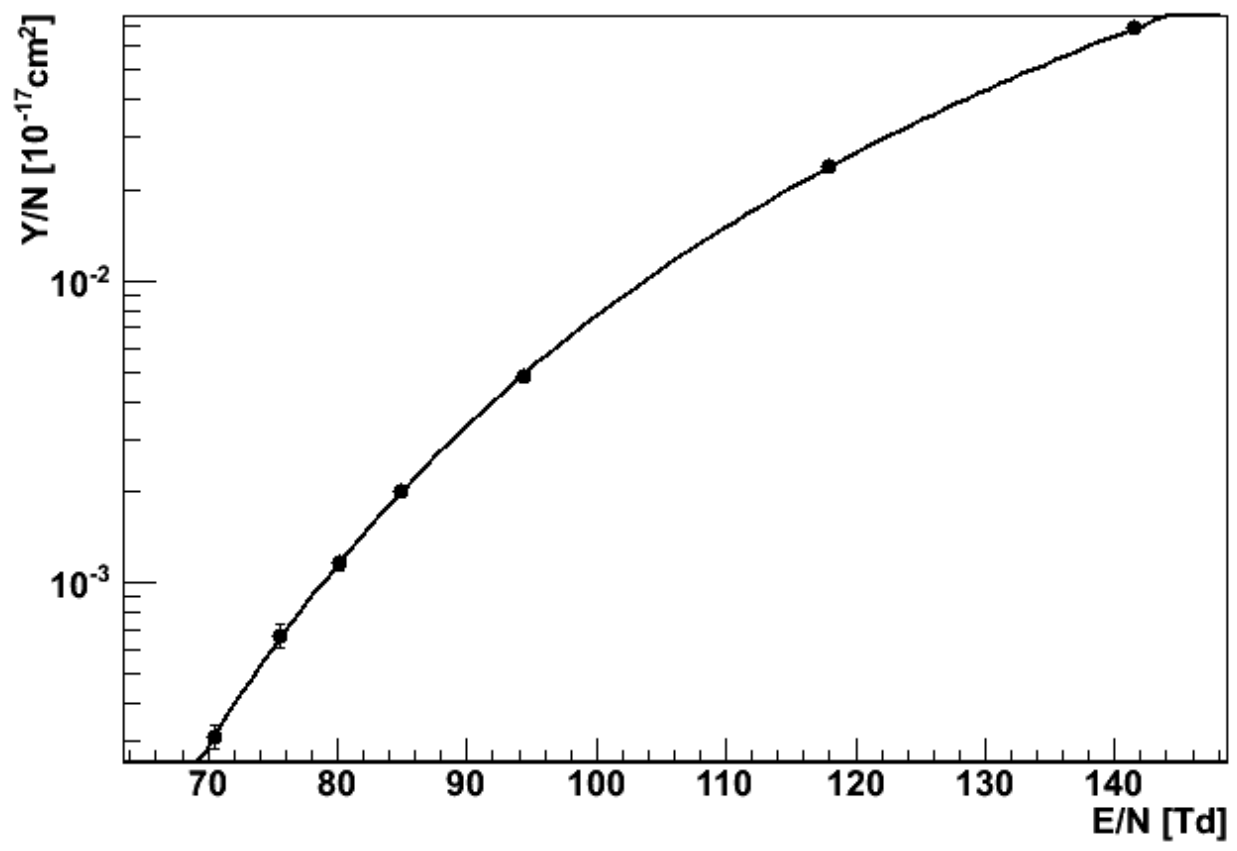

Figure 5. The reduced excitation photon yield as a function of the reduced electric field strength $(1 \mathrm{Td}=$ $10^{-17} \mathrm{Vcm}^{2}$ ). The line displays the empirical fit to the calculated points (statistical uncertainty only) by the third order polynomial from table 3.

Fig. 6 presents luminescence predictions from the simulation in terms of the mean free path of electrons as a function of applied electric field strength. We calculate the mean free path as the scale parameter for an exponential probability distribution which describes the emission of a single excitation photon due to inelastic electron argon scattering in liquid argon. The results suggest that fields in excess of $3 \mathrm{GV} / \mathrm{m}$ are required in order to trigger any significant amount of photon emission on millimetre distance scales.

\section{Conclusion}

A Monte-Carlo simulation of electron transport in liquid argon has been implemented based on interaction cross-sections from experimental measurements, where they exist, and theoretically 


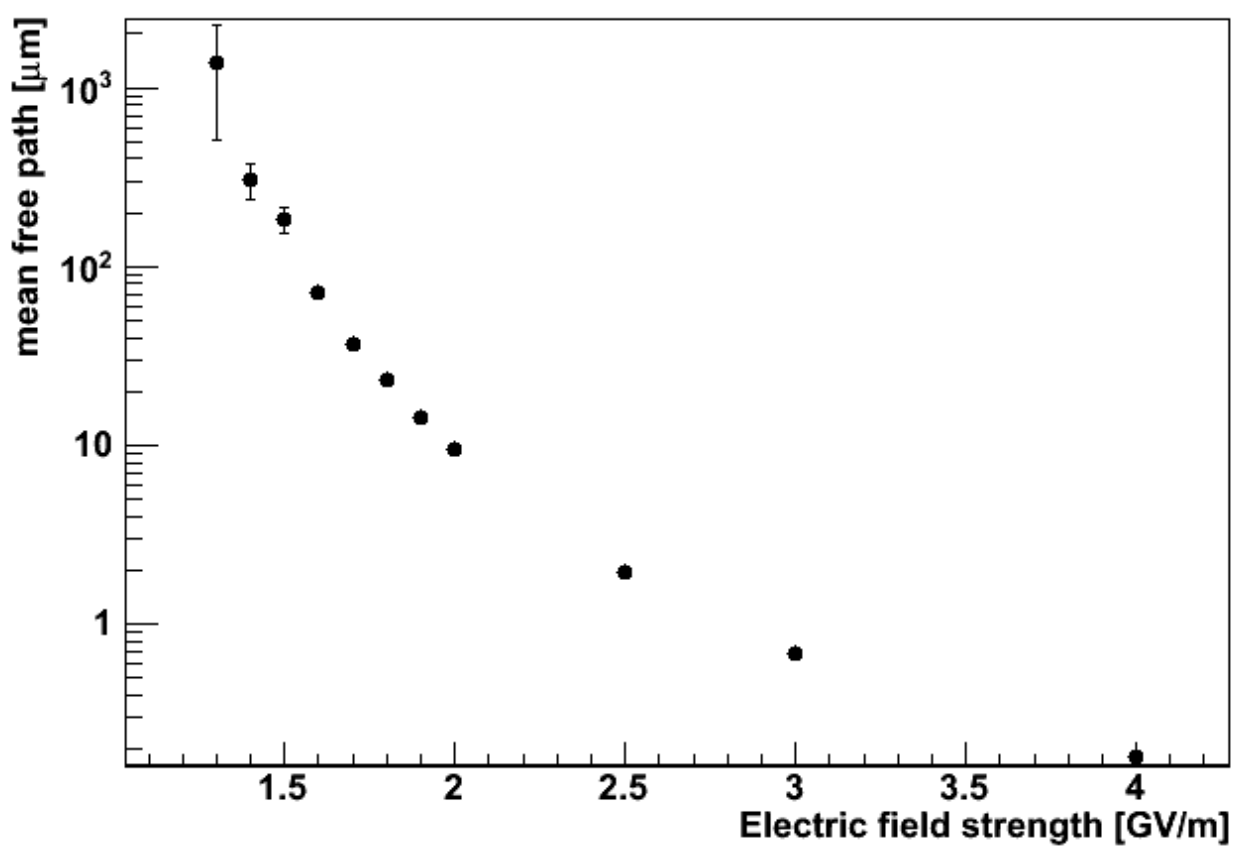

Figure 6. Shown is the mean free path length (statistical uncertainty only) for electrons in liquid argon as function of electric field strength as discussed in the text.

motivated estimates from the literature where they do not. Resulting predictions of the model for drift velocity and transverse diffusion are consistent with existing measurements.

Results on electroluminescence in liquid argon suggest that very large electric fields (i.e. in excess of $1 \mathrm{GV} / \mathrm{m}$ ) would be needed in order to observe any significant photon yield. The observation of electroluminescence in THGEM holes [四] suggests that these huge field strengths are present at some points inside the holes of THGEM structures. Given that the applied voltage gradient across the THGEM holes in [䧃] was only about $6 \times 10^{6} \mathrm{~V} / \mathrm{m}$, it could be that the larger fields needed for electroluminescence were only achieved locally around microscopic spurs or protrusions caused by the drilling procedure used to produce the holes. Further experimental investigations are currently underway to answer this question. The Monte-Carlo tool introduced in this publication should enable us to build a satisfactory model of the observations made in [1] and generally of measurements based on electron transport in liquid argon.

\section{Acknowledgments}

This research was partly funded by STFC and the RDF scheme of the University of Warwick.

\section{References}

[1] P.K. Lightfoot, G.J. Barker, K. Mavrokoridis, Y.A. Ramachers and N.J.C. Spooner,2009 JINST 4 P04002 
[2] S. Amerio, et al., Design, construction and tests of the ICARUS T600 detector, Nucl. Instr. and Meth.

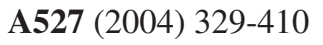

[3] A. Rubbia, J. Phys., Conf. Ser. 171 (2009) 012020

[4] B. Baibussinov et al., arXiv:0704.1422

[5] A. Rubbia, hep-ph/0402110

[6] L. Bartoszek et al., hep-ex/0408121

[7] D.B. Cline et al., astro-ph/0105442

[8] ArgoNeuT web-page

[9] A. Ereditato and A. Rubbia, Nucl. Phys. B (Proc. Suppl.) 154 (2006) 163

[10] A. Meregaglia, Nucl. Phys. B (Proc. Suppl.) 159(2006) 101

[11] T. Abe et al., 2009 JINST 4 T05001

[12] A. Breskin et al., Nucl. Instr. and Meth. A598 (2009) 107

[13] P.K. Lightfoot, G.J. Barker, K. Mavrokoridis, Y.A. Ramachers and N.J.C. Spooner, 2008 JINST 3 P10001

[14] T.H.V.T. Dias, A.D. Stauffer and C.A.N. Conde, J. Phys. D: Appl. Phys. 19 (1986) 527

[15] E. Aprile, A.E. Bolotnikov, A.I. Bolozdynya and T. Doke, Noble Gas Detectors, Wiley-VCH, Weinheim, 2006

[16] M. Wojcik and M. Tachiya, Chem. Phys. Lett. A363 (2002) 381

[17] V.M. Atrazhev and E.G. Dmitriev, J.Phys. C: Sol. State Phys. 18 (1985) 1205

[18] H.R. Skullerud, J. Phys. D: Appl. Phys. 1 (1968) 1567

[19] C. Jacoboni and L. Reggiani, Rev. Mod. Phys. 55(1983) 645

[20] E.E. Kunhardt, Phys. Rev. B 44 (1991) 4235

[21] Y. Sakai, K. Sukegawa, S. Nakamura and H. Tagashira, IEEE Trans. Electr. Insul. 23 (1988) 609

[22] S. Nakamura, Y. Sakai and H. Tagashira, Chem. Phys. Lett. 130 (1986) 551

[23] S.L. Lin and J.N. Bardsley, J. Chem. Phys.66 (1977) 435

[24] J. Lekner, Phys. Rev. 158 (1967) 130

[25] M.H. Cohen and J. Lekner, Phys. Rev. 158 (1967) 305

[26] V.M. Atrazhev and I.T. Iakubov, J. Phys. C: Sol. State Phys. 14 (1981) 5139

[27] A.D. Stauffer, T.H.V.T. Dias and C.A.N. Conde, Nucl. Instr. and Meth. A242 (1986) 327

[28] N.J. Mason and W.R. Newell, J. Phys. B: At. Mol. Phys. 20 (1987) 1357

[29] W. Walkowiak, Nucl. Instr. and Meth. A449 (2000) 288

[30] L. S. Miller, S. Howe, and W. E. Spear, Phys. Rev. 166(1968) 871

[31] E. Shibamura, T. Takahashi, S. Kubota and T. Doke, Phys. Rev. A 20(1979) 2547

[32] V.M. Atrazhev and I.V. Timoshkin, IEEE Trans. Dielectr. Electr. Insul. 5 (1998) 450

[33] K. Koura, Australian J. Phys. 40 (1987) 61

[34] C.M.B. Monteiro, et al., Secondary scintillation yield in pure argon, Phys. Lett. B668 (2008) 167 